\title{
Estudo da relação entre a Atividade Física e a função respirató- ria: análise da composição corporal e dos valores espirométricos de alunos Portugueses e Italianos
}

\section{Relationship between Physical Activity and respiratory function: analysis of corporal composition and spirometric values of Portuguese and Italian students}

\author{
Rui Miguel Paulo1*, João Manuel Petrica ${ }^{1}$, Júlio Cardoso Martins ${ }^{2}$, Filippo Pichetto ${ }^{3}$, Fla- \\ vio Alberto Faure-Rolland ${ }^{3}$, Francesca Magno $^{3}$
}

ARTIGO ORIGINAL | ORIGINAL ARTICLE

\begin{abstract}
Objetivou-se analisar a influência e a relação da atividade física (AF) na composição corporal e nos valores espirométricos, relacionando esses indicadores com a função respiratória. Amostra com 179 indivíduos (85 Italianos; 94 Portugueses) alunos do ensino superior, dividida em três grupos: GESC+EXERC - 64 alunos com AF supervisionada, curricular e extracurricular, mínimo 4 sessões/semana; GESCOLA - 66 alunos com AF supervisionada, curricular, mínimo 2 sessões/semana; GSEDENTÁRIOs - 49 alunos sedentários. Aplicou-se uma adaptação do questionário de Telama et al. (1997), avaliou-se a espirometria através do espirómetro Microquark/Cosmed, o IMC e perímetro da cintura (PC). Após a verificação da normalidade da amostra (Kolmogorov-Smirnov), utilizou-se a Análise de variância e o teste não paramétrico MannWhitney. Utilizou-se ainda o teste de correlação de Spearman. O GESC+EXERC dos dois países obteve me-

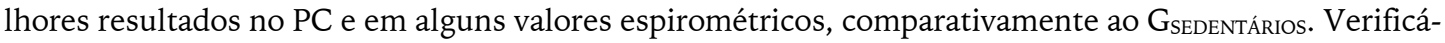
mos na amostra uma correlação positiva entre o IMC e o PC, e para os alunos Portugueses correlação negativa entre a composição corporal e alguns valores espirométricos. A Itália apresenta melhores indicadores de composição corporal, enquanto Portugal os apresenta na espirometria. Há evidências que os alunos que praticam exercício supervisionado apresentaram melhores indicadores de composição corporal e na função respiratória.

Palavras-chaves: Atividade Física, Função respiratória, Composição Corporal, Espirometria
\end{abstract}

\begin{abstract}
The objective was to analyze the influence and relationship of physical activity (PA) on corporal composition and on spirometric values, associating these indicators with the respiratory function. Sample with 179 individuals (85 Italians; 94 Portuguese) who were higher education students, and they were divided into three groups: $\mathrm{G}_{\mathrm{SCH}+\mathrm{EXER}}-64$ students doing supervised exercise, curricular and extra-curricular, at least 4 sessions/week; $\mathrm{G}_{\mathrm{SCHOOL}}-66$ students doing supervised curricular PA, at least 2 sessions/week; $\mathrm{G}_{\text {SEDENTARY }}-49$ sedentary students. We applied an adaptation of the questionnaire Telama et al. (1997), and measured the spirometry values through the spirometer Microquark/Cosmed, the BMI and Waist Circumference (WC). After checking the normality of the sample (Kolmogorov-Smirnov), we used the ANOVA and the nonparametric Mann-Whitney test. Was also used the Spearman correlation test. The GSCH-EXER of both nationalities achieved significantly better WC and some spirometric values, compared to GSEDENTARY. We confirmed in the sample there was a positive correlation between BMI and WC, and for Portuguese students a negative correlation between body composition and some spirometric values. Italians had better corporal composition values, while the Portuguese had better spirometric values. The evidence was that the students who did supervised exercise had better corporal composition and respiratory function.

Keywords: Physical Activity, Respiratory Function, Corporal Composition, Spirometry
\end{abstract}

Artigo recebido a 17.07.2013; Aceite a 17.06.2014

${ }^{1}$ Escola Superior de Educação do Instituto Politécnico de Castelo Branco, Portugal

${ }^{2}$ Universidade da Beira Interior, Covilhã, Portugal

${ }^{3}$ Scuola Universitaria Interfacoltà in Scienze Motorie, Torino, Italia

* Autor correspondente: Escola Superior de Educação, Rua Prof. Dr. Faria de Vasconcelos, 6000-266 Castelo Branco, Portugal E-mail: ruipaulo@ipcb.pt 


\section{INTRODUÇÃO}

A literatura científica é unânime em considerar que a prática regular de atividade física $(\mathrm{AF})$ proporciona efeitos positivos sobre o organismo, nos diversos órgãos e sistemas (Dias et al., 2008), e que, por sua vez, a inatividade física/sedentarismo tem influência no sobrepeso e obesidade (Padez, Fernandes, Mourão, Moreira, \& Rosado, 2004), podendo provocar uma síndrome restritiva, pelo acumular de gordura peritorácica e abdominal, podendo originar diminuição dos volumes pulmonares (He et al., 2009; Silva, Boin, Pareja, \& Magna, 2007).

Mesmo sendo um prognosticador débil da gordura corporal, o Índice de Massa Corporal (IMC) é bastante útil devido à sua relação direta com vários indicadores de saúde, ou seja, à medida que aumenta o IMC, aumenta também o risco de complicações cardiovasculares, alguns cancros, diabetes mellitus, osteoartrite e doença renal, daí a sua importância em termos epidemiológicos (McArdle, Katch, \& Katch, 2011). Por sua vez, o perímetro da cintura (PC) permite avaliar a distribuição central da gordura corporal, sendo esta medida importante na avaliação do risco cardiovascular, pelo facto de ser forte preditora da quantidade de gordura visceral, principal responsável pelo aparecimento de alterações metabólicas e de doenças cardiovasculares (Pereira, Sichieri, \& Marins, 1999; Rizzo, Ruiz, Hurtig-Wennlöf, Ortega, \& Sjöström, 2007). O sobrepeso e a obesidade estão também relacionados com o aumento do risco da disfunção respiratória, sendo que a prevalência dos sintomas aumenta, quanto maior for o IMC e o PC (Sahebjami, 1998).

A espirometria é o exame que mensura capacidades e débitos pulmonares, a partir de manobras respiratórias padronizadas, comparando-os com padrões de referência para a altura, o sexo, a idade e estados diferenciados de saúde, como o perfil tabágico (Rodrigues et al., 2002). Averigua a existência de obstrução ao débito de ar, ou seja, se as vias aéreas têm alguma anormalidade, ou se o volume dos pulmões está normal.

Diversos estudos que relacionam o desempenho respiratório, em indivíduos com idades e hábitos díspares, e a prevalência de sobrepeso e obesidade (Chinn, 2005; Rubinstein, Zamel, DuBarry, \& Hoffstein, 1990) consideram ter identificado alterações ao nível do sistema respiratório, especialmente a redução dos volumes e capacidades pulmonares: volume de reserva expiratória, volume expiratório forçado no primeiro segundo $\left(\mathrm{VEF}_{1}\right)$, capacidade vital forçada (CVF) e das taxas de débito expiratório.

As complicações respiratórias como a intolerância ao exercício, a asma e a apneia do sono são constantes em adolescentes e jovens obesos, podendo limitar a prática de AF e desportiva, dificultando a perda de peso (Chinn, 2005; de Sá Pinto, de Barros Holanda, Radu, Villares, \& Lima, 2006). Com o aumento da deposição de gordura na cavidade torácica e na cavidade abdominal, progressivas alterações podem ocorrer na função respiratória (Fung, Lau, Chow, Lee, \& Wong, 1990). Tais modificações nesta função são mais comuns na obesidade central, em que a acumulação de tecido adiposo se localiza, principalmente, na região da cintura (Collins, Hoberty, Walker, Fletcher, \& Peiris, 1995; Sue, 1997).

A gordura armazenada na cavidade abdominal, designada de ginóide, exerce, provavelmente, um efeito mecânico direto na caixa torácica e no diafragma, por um mecanismo de compressão, que, por sua vez, limita a expansibilidade pulmonar, causando diminuição dos volumes pulmonares (Paulo, Petrica, \& Martins, 2013; Sue, 1997). Desta forma, a obesidade e o padrão de distribuição da gordura corporal podem influenciar os resultados da função respiratória (Lazarus, Sparrow, \& Weiss, 1997).

Deste modo, a avaliação dos valores espirométricos representa uma ferramenta essencial para o diagnóstico de patologias pulmonares. A CVF é o volume eliminado em manobra expiratória forçada desde a capacidade pulmonar total até ao volume residual. A CVF é um teste importante porque, durante a expiração, um indivíduo pode atingir o limite do débito máximo, mas, como a curva define o limite para o débito, ela é altamente reprodutível e, mais importante, o débito máximo é muito sensível na maioria 
das patologias comuns que afetam o pulmão (Salas et al., 2011). Outro volume avaliado e de extrema importância é o $\mathrm{VEF}_{1}$, que corresponde à quantidade de ar eliminada no primeiro segundo da manobra expiratória forçada. Também o Débito Expiratório Máximo Instantâneo (DEMI) é um indicador importante da espirometria.

A presente investigação teve como principal objetivo averiguar qual a influência e a relação da AF na composição corporal (IMC e PC) e nos valores espirométricos (CVF, DEMI e $\mathrm{VEF}_{1}$ ), verificando se há correlação entre os valores da composição corporal e os volumes pulmonares dos sujeitos da amostra, investigando as diferenças entre os dois países.

\section{Amostra}

\section{MÉTODO}

A amostra foi composta por 179 indivíduos voluntários de ambos os sexos (85 Italianos e 94 Portugueses), aparentemente saudáveis, com idades compreendidas entre os 18 e os 31 anos, com uma média de idades de 21.94 anos (DP \pm 2.56). Todos os sujeitos da amostra são alunos do ensino superior público, em Itália e em Portugal. A amostra foi selecionada por conveniência, mas foram estabelecidos, em ambos os países, os mesmos critérios de inclusão a fim de se selecionarem os sujeitos. Todos os indivíduos tinham que ser alunos do Ensino Superior público, tinham que preencher os requisitos para um dos três grupos da amostra, não apresentar contraindicações para a prática de exercício físico e consentimento positivo para a participação no estudo (incluindo a realização de todas as avaliações).

Neste estudo, a amostra foi dividida em três grupos, por país, de acordo com critérios subja-

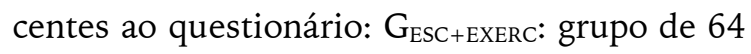
alunos (22 Italianos e 42 Portugueses), praticantes de AF e desportiva com exercício supervisionado (programa multicomponente) com intensidade periodizada, participantes de atividades letivas (aulas práticas de distintas modalidades) e extracurriculares (clubes, ginásios, entre outros), com 4 sessões/semana no mí-

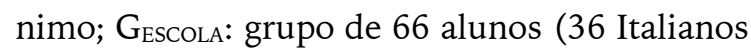
e 30 Portugueses) praticantes de AF e desportiva, participantes de atividades letivas, com exercício supervisionado com intensidade periodizada, com 2 sessões/semana no mínimo; $\mathrm{G}_{\mathrm{SE}-}$ DENTÁrios: grupo de 49 alunos (27 Italianos e 22 Portugueses) sedentários (tabela 1).

\section{Instrumentos}

\section{Questionário de $A F$}

Aplicou-se uma adaptação de um questionário validado por Telama, Yang, Laakso, e Viikari (1997) que apenas teve como objetivo constituir critério para a divisão e seleção da amostra, pelos diferentes grupos, e não recolher dados fulcrais para as variáveis dependentes, facto que levou a que não fosse necessária a sua validação, para este estudo.

\section{Estatura}

Os valores de estatura foram mensurados em metros com aproximação aos milímetros através de um estadiómetro SECA (Germany, Hamburg), considerando o plano de referência do solo e o vértex, atendendo às padronizações sugeridas por Gordon, Chumlea e Roche (1991).

\section{Massa corporal}

A massa corporal foi medida em $\mathrm{Kg}$, através de uma balança digital, SECA 708 (Germany, Hamburg) com aproximação às centésimas, estando os sujeitos descalços com roupa leve (Gordon et al., 1991).

Para o IMC $(\mathrm{kg} / \mathrm{m} 2)$, as classificações utilizadas foram as da World Health Organization (1998).

\section{Perímetro da cintura}

Relativamente ao PC, os valores foram mensurados com uma fita Métrica (Rosscraft) em fibra de vidro, de dois metros, e com resolução de $1 \mathrm{~mm}$, de acordo com procedimentos recomendados (Callaway et al., 1988). 
Tabela 1

Caraterização da amostra $(n=179)$ e constituição dos grupos

\begin{tabular}{|c|c|c|c|c|c|c|}
\hline & \multicolumn{3}{|c|}{ ALUNOS ITALIANOS } & \multicolumn{3}{|c|}{ ALUNOS PORTUGUESES } \\
\hline & Mínima & Máxima & Média \pm DP & Mínima & Máxima & Média \pm DP \\
\hline Idade (anos) & 19 & 30 & $22.60 \pm 2.60$ & 18 & 31 & $21.35 \pm 2.38$ \\
\hline $\operatorname{IMC}\left(\mathrm{kg} / \mathrm{m}^{2}\right)$ & 17.8 & 38.6 & $22.30 \pm 3.28$ & 18.2 & 34.1 & $23.98 \pm 2.71$ \\
\hline $\mathrm{PC}(\mathrm{cm})$ & 57 & 115 & $75.17 \pm 9.10$ & 65 & 104 & $79.26 \pm 7.58$ \\
\hline Índice Cint/Anca & .63 & 1,03 & $.82 \pm .70$ & .65 & .94 & $.83 \pm .07$ \\
\hline Glicem. mg/dl & 65 & 126 & $88.89 \pm 9.85$ & 59 & 97 & $80.03 \pm 6.57$ \\
\hline Triglic. mg/dl & 56 & 301 & $98.84 \pm 47.47$ & 154 & 271 & $182.86 \pm 24.82$ \\
\hline Colest. mg/dl & 112 & 264 & $167.56 \pm 29.95$ & 71 & 261 & $122.56 \pm 52.09$ \\
\hline PAS mmHg & 93 & 155 & $122.05 \pm 13.02$ & 83 & 148 & $120.20 \pm 11.48$ \\
\hline PAD mmHg & 50 & 95 & $73.51 \pm 8.30$ & 47 & 100 & $65.65 \pm 8.81$ \\
\hline $\mathrm{FC}_{\text {repouso }} \mathrm{Bpm}$ & 42 & 111 & $70.55 \pm 14.57$ & 45 & 85 & $63.95 \pm 11.62$ \\
\hline CVF \% Prev. & 60 & 115 & $90.55 \pm 9.14$ & 79 & 115 & $97.85 \pm 9.71$ \\
\hline PFE \% Prev. & 43 & 145 & $95.66 \pm 21.97$ & 63 & 144 & $106.23 \pm 16.90$ \\
\hline $\mathrm{VEF}_{1} \%$ Prev. & 58 & 133 & $93.89 \pm 12.28$ & 74 & 119 & $100.05 \pm 11.10$ \\
\hline Grupos & $\mathbf{N}$ & $\begin{array}{l}\text { Média } \pm \text { DP } \\
\text { Idade (anos) }\end{array}$ & \multicolumn{2}{|c|}{$\begin{array}{c}\text { Masculino } \\
\mathrm{n}\end{array}$} & \multicolumn{2}{|c|}{$\begin{array}{c}\text { Feminino } \\
\mathbf{n}\end{array}$} \\
\hline GsEDENTÁRIOS_ITÁLIA & 27 & \multicolumn{2}{|l|}{$22.15 \pm 2.57$} & 10 & \multicolumn{2}{|r|}{17} \\
\hline Gescola_tTálIA & 36 & \multicolumn{2}{|l|}{$23.14 \pm 2.82$} & 19 & \multicolumn{2}{|r|}{17} \\
\hline GESC+EXERC_tT́́LIA & 22 & \multicolumn{2}{|l|}{$22.27 \pm 2.19$} & & \multicolumn{2}{|r|}{4} \\
\hline GsEDENTÁRIOS_PORTUGAL & 22 & $21.23 \pm 1.80$ & \multicolumn{2}{|c|}{$\begin{array}{c}18 \\
7\end{array}$} & \multicolumn{2}{|r|}{15} \\
\hline GESCOLA_PORTUGAL & 30 & $20.87 \pm 1.33$ & \multicolumn{2}{|c|}{16} & \multicolumn{2}{|r|}{14} \\
\hline GESC+EXERC_PORTUGAL & 42 & $21.76 \pm 3.09$ & \multicolumn{2}{|c|}{32} & & 10 \\
\hline TOTAL & 179 & $21.94 \pm 2.56$ & & & & 77 \\
\hline
\end{tabular}

\section{Espirometria}

Finalmente, para a mensuração dos valores espirométricos (DEMI, $\mathrm{VEF}_{1}$ e CVF), recorreuse ao espirómetro Microquark da Cosmed. A espirometria é um teste que auxilia no diagnóstico, na prevenção e na quantificação dos distúrbios ventilatórios, sendo realizada durante uma manobra expiratória forçada. Pela sua complexidade, a sua realização exige a compreensão e colaboração do paciente, equipamento calibrado (através de seringa apropriada) e utilização de técnicas padronizadas empregadas por pessoal especializado. Os valores obtidos devem ser comparados aos previstos para determinado grupo populacional e a sua interpretação feita à luz dos dados clínicos e epidemiológicos. O espirómetro mensura o volume de ar expirado, especialmente útil na análise dos dados derivados da manobra expiratória forçada, seguindo o pro- tocolo de acordo com as recomendações de Miller et al. (2005). Os sujeitos da amostra apresentavam caraterísticas clínicas, aparentemente saudáveis, tendo sido devidamente selecionados os parâmetros do programa da Cosmed.

\section{Procedimentos}

Este estudo foi aprovado pela Comissão de Ética da Faculdade de Ciências da Saúde da Universidade da Beira Interior, Covilhã, Portugal. Foi obtida autorização por escrito dos sujeitos avaliados, por meio do termo de consentimento livre e informado, sendo estes antecipadamente informados do âmbito e objetivos do estudo, bem como da salvaguarda dos dados individuais.

Todas as instruções relativas aos procedimentos foram apresentadas para que cada sujeito recebesse as mesmas indicações. Foram 
respeitadas as normas internacionais de experimentação com humanos (Declaração de Helsínquia, 1975).

Os critérios subjacentes à seleção dos sujeitos dos dois grupos estudados eram comparáveis nas suas principais variáveis socioeconómicas e biológicas (confirmado com a aplicação do questionário), além dos testes terem sido aplicados com a mesma técnica e os mesmos aplicadores.

\section{Análise Estatística}

Relativamente aos procedimentos estatísticos, recorrendo ao Software SPSS 19.0, na primeira análise, procedeu-se à verificação da normalidade da amostra (Kolmogorov-Smirnov). Para a variável que apresentou distribuição normal $\left(\mathrm{VEF}_{1}\right)$, utilizou-se a Análise de variância (Anova), através do teste LSD. Para as restantes variáveis, que não apresentaram distribuição normal (IMC, PC, CVF e DEMI), procedeu-se à utilização do teste Mann-Whitney.

Para as análises da correlação, utilizámos o teste de correlação não-paramétrico de Spearman. Adotou-se para ambas as análises um nível de significância de $5 \%$.

\section{RESULTADOS}

Para a análise entre as variáveis categóricas, são apresentados, numa primeira fase, os resultados através da média e desvio padrão. Na tabela 2, e numa primeira análise, verifica-se que o G GSC+EXERC_PORTUGAL apresenta valores médios absolutos mais favoráveis em todos os indicadores avaliados comparativamente ao GESCOLA_PORTUGAL, o mesmo se verificando na comparação com o GsEDENTÁRIOS_PORTUGAL, à exceção da CVF, que é semelhante.

Relativamente ao GESC+EXERC_tTÁLIA, este apenas apresenta valores mais favoráveis ao nível dos valores espirométricos, comparativamente ao Gsedentários_ttália e ao Gescola_ttália, porque apresenta valores mais elevados em relação aos indicadores de composição corporal, principalmente no PC.

Na comparação dos 3 grupos de cada país, entre si, podemos observar na tabela 3 que, ao nível da composição corporal, para o IMC e o $\mathrm{PC}$, quer os Portugueses, quer os Italianos apresentam diferenças estatisticamente significativas $(p \geq .05)$ e muito significativas $(p \geq .01)$ entre o GsEDENTÁrIos e o G GSC+EXERC, apesar de no caso dos alunos Italianos, serem os sedentários a apresentar os melhores resultados para o IMC. Já para os valores espirométricos avaliados, podemos verificar que, também aqui, para a $\mathrm{VEF}_{1}$ e o DEMI, ambos os países apresentam diferenças estatisticamente significativas $(p \geq .05)$ entre o $\mathrm{G}_{\text {ESC+EXERC }}$ comparativamente ao $\mathrm{G}_{\text {SEDENTÁ- }}$ Rios e ao Gescola. Relativamente à CVF, os dois países apresentam diferenças estatisticamente significativas na comparação entre o $\mathrm{G}_{\text {ESC+EXERC }}$ e o G GEDENTÁRIOS.

Para a comparação entre os grupos homólogos dos dois países, podemos verificar que, para a composição corporal (IMC e PC), apenas o $\mathrm{G}_{\text {ESC+EXERC }}$ apresenta valores semelhantes. Quer o $\mathrm{G}_{\text {SEDENTÁRIOS, }}$ quer o $\mathrm{G}_{\text {ESCOLA, }}$ apresentam diferenças estatisticamente significativas $(p \geq .05)$ e muito significativas $(p \geq .01)$ para este indicador. Nesta comparação, os alunos Italianos apresentam os resultados aparentemente mais favoráveis, com valores de IMC e PC mais baixos.

Relativamente aos valores espirométricos avaliados, para a CVF, verificamos diferenças estatisticamente significativas entre os 3 grupos dos dois países, manifestando os alunos Portugueses resultados conducentes a indicadores mais favoráveis. Para os outros indicadores (DEMI e $\mathrm{VEF}_{1}$ ), apenas se verificam diferenças estatisticamente significativas entre o $G_{\text {ESCOLA, }}$ sendo também os alunos Portugueses a revelar resultados aparentemente mais favoráveis. 
8 | RM Paulo, JM Petrica, JC Martins, F Pichetto, FA Faure-Rolland, F Magno

Tabela 2

Média e desvio padrão dos resultados das variáveis avaliadas, dividida pelos grupos

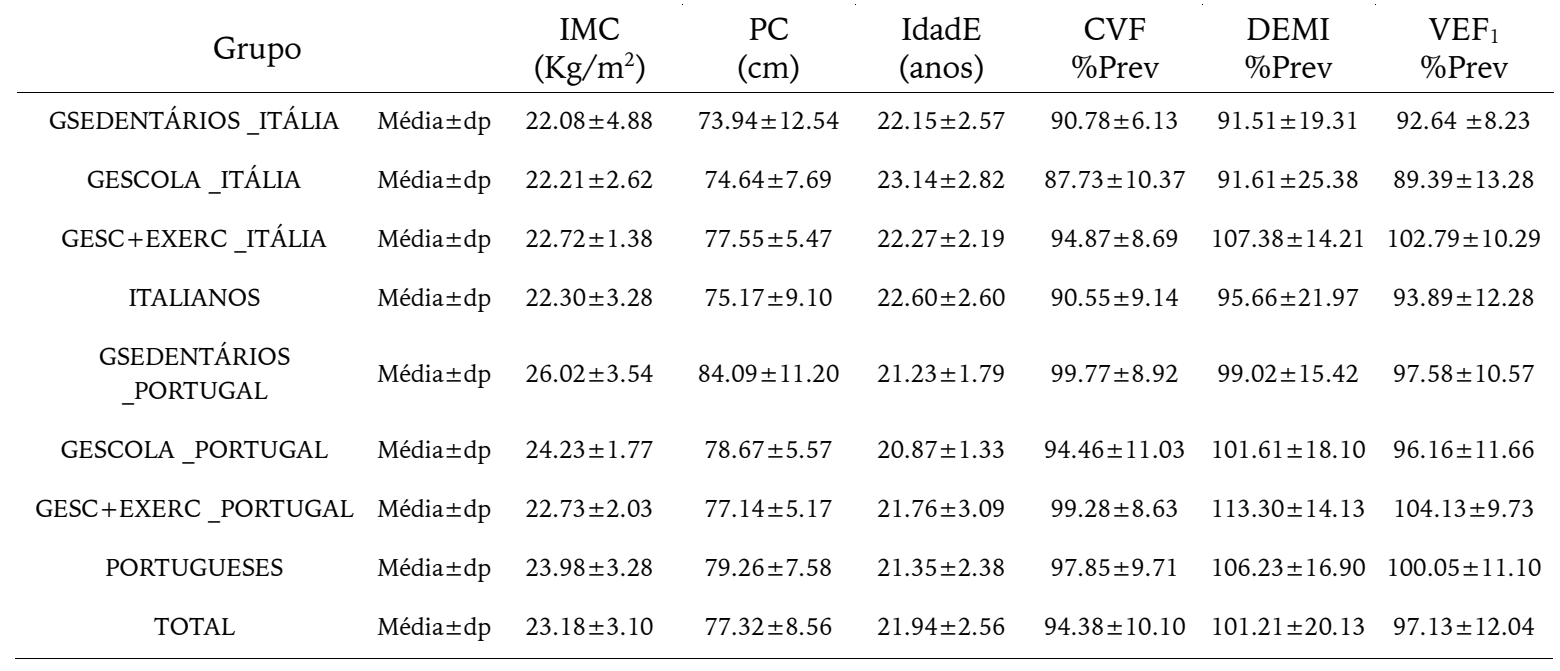

\section{Correlação}

Um dos objetivos deste estudo pretendia verificar a correlação entre as variáveis de estudo. Desta forma, podemos observar na tabela 4 que, quer para os alunos Portugueses, quer para os alunos Italianos, em todos os grupos, verifica-se uma correlação positiva muito significativa estatisticamente $(p \leq .01)$ entre o IMC e o PC, com coeficientes de correlação elevados. Desta forma, à medida que aumenta o IMC, há também uma tendência para aumentar o PC.

Tabela 3

Nível de significância das comparaçôes entre grupos, para as variáveis IMC, PC, CVF, DEMI e VEF

\begin{tabular}{|c|c|c|c|c|c|c|c|}
\hline (I) Grupo & (J) Grupo & $\begin{array}{c}\text { IDADE } \\
p\end{array}$ & $\begin{array}{c}\mathrm{IMC} \\
p\end{array}$ & $\begin{array}{c}\mathrm{PC} \\
p\end{array}$ & $\begin{array}{c}\text { CVF } \\
p\end{array}$ & $\begin{array}{c}\text { DEMI } \\
p\end{array}$ & $\begin{array}{c}\mathrm{VEF}_{1} \\
p\end{array}$ \\
\hline GSEDENTÁRIOS_ITÁLIA & Gescola_tTÁLIA & .166 & .104 & .120 & .246 & .632 & .241 \\
\hline GsEDENTÁRIOS_TTÁLIA & GESC+EXERC_tTÁLIA & .708 & $.008^{* *}$ & $.007^{* *}$ & .172 & $.003^{* *}$ & $.001^{* *}$ \\
\hline Gescola_tTÁLIA & GESC+EXERC__tT́́LIA & .275 & .109 & .073 & $.018^{*}$ & $.005^{* *}$ & $.000^{* *}$ \\
\hline GsEDENTÁRIOS_PORTUGAL & Gescola_poRtugal & .473 & .182 & .114 & $.042^{*}$ & .795 & .641 \\
\hline GsEDENTÁRIOS_PORTUGAL & GESC+EXERC_PORTUGAL & .954 & $.000^{* *}$ & $.013^{*}$ & .865 & $.001^{* *}$ & $.023^{*}$ \\
\hline Gescola_poRtugal & GESC+EXERC_PORTUGAL & .456 & $.002^{* *}$ & .409 & $.027^{*}$ & $.001^{* *}$ & $.002^{* *}$ \\
\hline GSEDENTÁRIOS_ITÁLIA & GSEDENTÁRIOS_PORTUGAL & .324 & $.000^{* *}$ & $.001^{* *}$ & $.000^{* *}$ & .260 & .115 \\
\hline GesCola_ITÁLIA & Gescola_portugal & $.000^{* *}$ & $.000^{* *}$ & $.013^{*}$ & $.039^{*}$ & $.043^{*}$ & $.012^{*}$ \\
\hline GESC+EXERC_ITÁLIA & GESC+EXERC_PORTUGAL & .109 & .611 & .921 & $.033^{*}$ & .073 & .639 \\
\hline ITALIANOS & PORTUGUESES & $.000^{* *}$ & $.000^{* *}$ & $.000^{* *}$ & $.000^{* *}$ & .001 & $.001^{* *}$ \\
\hline
\end{tabular}

${ }^{*} \mathrm{p}<.05,{ }^{* *} \mathrm{p}<.01$

Também na mesma tabela, relativamente às restantes variáveis, para os grupos de Portugal, observamos que apenas se verificam correlações negativas, estatisticamente significativas $(p \leq$
.05) entre o IMC e a CVF para o G $\mathrm{ESSOla}_{\text {P PORTUGAL }}$ e entre o PC e o DEMI para o GSEDENTÁRIOS_PORTUGAL. Assim, para estes grupos, à medida que au- 
menta o IMC e o PC, há uma tendência para diminuírem os valores da CVF e DEMI, respetivamente. Verificamos ainda uma correlação positiva, estatisticamente significativa $(p \leq .01)$, entre o IMC e a CVF, para o GESC+EXERC_PORTUGAL.

Podemos ainda verificar na tabela 4 que, relativamente aos alunos Italianos, há uma correlação positiva, estatisticamente significativa ( $p$ $\leq .05)$, entre o IMC e a CVF e entre o IMC e o
DEMI, para o Gescola_trália. Ainda no mesmo grupo verificamos uma correlação positiva, estatisticamente significativa $(p \leq .01)$, entre o IMC e o VEF 1 . Verificamos também uma correlação positiva, na mesma ordem de grandeza, entre o IMC e o DEMI, para o G GSC-EXERC_tTálIA. Para os grupos de Itália o PC não ostenta correlação com nenhuma variável espirométrica.

Tabela 4

Nível de significância das correlaçôes e coeficiente de correlação entre as variáveis IMC, PC, CVF, DEMI e VEF, para os elementos da amostra, divididos pelos países e pelos grupos

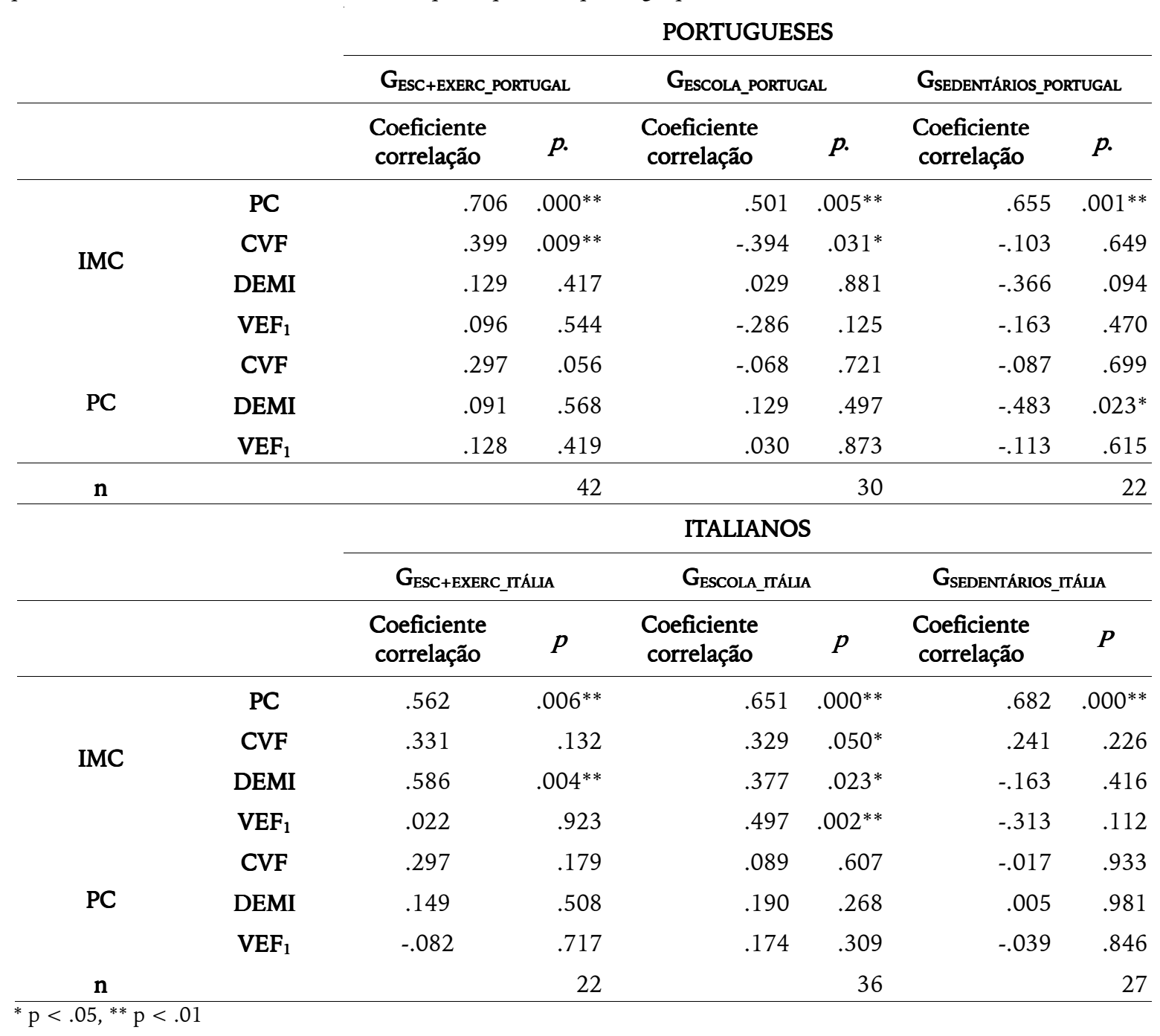

\section{DISCUSSÃO}

Um dos objetivos do presente estudo pretendeu verificar se a AF supervisionada e planificada poderá estar relacionada, influenciando a composição corporal (IMC e PC) e os valores espirométricos (CVF, DEMI e $\mathrm{VEF}_{1}$ ) dos alunos Portugueses e Italianos. Os resultados obtidos parecem reforçar a importância da prática da $\mathrm{AF}$ 
orientada e supervisionada, na manutenção e melhoria dos valores espirométricos e da composição corporal, comparando com atividades e estilos de vida sedentários (Chinn, 2005; de Sá Pinto et al., 2006; Dias et al., 2008; Padez et al., 2004; Paulo et al., 2013; Silva et al., 2007).

Relativamente à composição corporal, procurou-se verificar se os indivíduos mais ativos apresentavam valores inferiores e mais ajustados em relação aos sedentários, encontrando-se diferenças significativas e muito significativas em relação a algumas comparações entre os grupos, principalmente entre o $G_{E S C+E X E R C}$ e o $G_{S E-}$ DENTÁrios de cada país.

Na comparação entre os dois países, para os valores de composição corporal, verificámos que apenas o GESC+EXERC apresentou valores semelhantes. Para os outros dois grupos, os alunos Italianos apresentam valores mais favoráveis, estatisticamente aceitáveis. Uma explicação plausível será um maior gasto energético, no grupo dos praticantes de atividades físicas com prescrição planificada, sendo que também o tipo de alimentação e de hábitos de vida, determinados culturalmente, podem influenciar as diferenças. Apesar de a dieta alimentar não ter sido controlada nem prescrita aos sujeitos da amostra, sabemos que esta é um fator que pode influenciar alguns indicadores avaliados, pois, de acordo com Slentz et al. (2004), a combinação de uma dieta equilibrada e AF regular formam o meio mais efetivo do controlo do peso.

Relativamente aos valores espirométricos avaliados (CVF, DEMI e $V_{E F}$ ), o GESC+EXERC apresenta os melhores resultados, para os dois países, mostrando diferenças estatisticamente significativas em algumas comparações entre os grupos. Desta forma, parece que a prática de exercício físico supervisionado parece contribuir para a manutenção de valores espirométricos adequados, diminuindo assim o risco de patologias respiratórias (Sue, 1997). Na comparação entre os dois países, para os valores espirométricos avaliados, os alunos Portugueses apresentam os valores mais elevados e mais favoráveis, com diferenças acentuadas no $\mathrm{G}_{\mathrm{ESCOLA}}$, podendo, no entanto, acontecer que a fórmula de cálculo do valor predito seja influenciada, principalmente, pelas caraterísticas antropométricas dos sujeitos.

Verificámos uma correlação positiva, muito significativa, entre as variáveis da composição corporal, para os dois países. Desta forma, os nossos resultados indicam que, à medida que aumenta o IMC, o PC tem também tendência para aumentar, podendo indicar uma maior possibilidade de deposição de gordura na região abdominal, predizendo um eventual aumento da quantidade de gordura visceral, principal responsável pelo aparecimento patologias cardiovasculares (Paulo et al., 2013; Pereira et al., 1999; Rizzo et al., 2007).

No nosso estudo, para os alunos de ambos os países, não é evidente que o aumento do IMC e do PC contribuam de forma relevante para a diminuição dos valores espirométricos avaliados, isto porque não se verificou correlação negativa, aceitável estatisticamente, entre alguns dos valores, o que também se verificou em outros estudos (Dockery, Berkey, Ware, Speizer, \& Ferris, 1983; Fung et al., 1990). Mas, por outro lado, houve alguns desses valores espirométricos que revelaram correlação negativa com a composição corporal, mostrando assim, tal como noutros estudos (Chen, Horne, \& Dosman, 1993; Inselma, Milanese, \& Deurloo, 1993; Paulo et al., 2013), que as elevações dos valores de composição corporal (IMC e PC) poderiam acarretar diminuição na função respiratória. O sobrepeso e a obesidade estão também relacionados com o aumento do risco de sintomas respiratórios (Sahebjami, 1998), ou seja, a prevalência dos sintomas aumenta, quanto maior for o IMC ou a circunferência da cintura.

Distintos mecanismos têm sido propostos como possíveis efeitos da obesidade na função respiratória. As anomalias mais comummente referidas são a redução da capacidade residual funcional e do volume expiratório de reserva, devido à diminuição da expansibilidade da parede torácica, da compliance pulmonar e da maior resistência das vias aéreas (Zerah et al., 1993). 


\section{LIMITAÇÕES E SUGESTÕES}

Depois de uma reflexão exaustiva sobre os resultados e experiências retiradas da consecução deste estudo propomos algumas sugestões para futuros estudos, nesta área de investigação. Pensamos que, utilizando instrumentos semelhantes, os estudos longitudinais, com grupo de controlo e grupo(s) experimental(ais), com préteste e pós-teste, são mais efetivos, se o programa de exercício for ajustado, visto que este tipo de estudo (experimental), controla mais variáveis que podem influenciar os resultados, sendo as conclusões mais concretas.

Uma das problemáticas deste tipo de estudo passa pelas limitações ao nível da recolha de dados, mesmo depois de delimitar a amostra. Este tipo de estudo, com a aplicação destes instrumentos, com protocolos tão rígidos e demorados, levam a que muitos dos sujeitos, individualmente, ou aos que estão inseridos em instituições, muitas das vezes se recusem participar nas investigações, não dando consentimento. Outro problema, associado ao que acabamos de reportar, prende-se com o facto de que, para sujeitos menores de idade, ser "obrigatório" o preenchimento de um termo de consentimento informado, assinado pelo encarregado de educação. Fica, para este indicador, uma chamada de atenção para, no momento da seleção da amostra, ter em conta todos estes alvitres, para evitar constrangimentos.

Outro aspeto que seria interessante estudar, seria associar a estes parâmetros avaliados, alguns indicadores de aptidão física/capacidade funcional, com a aplicação de uma bateria de testes específica, visto que também surge a possibilidade de variar as idades dos sujeitos da amostra, o que levaria a aplicar testes de baterias validadas, de acordo com a faixa etária a eleger.

Finalmente, uma referência a um parâmetro de composição corporal utilizado neste estudo, o IMC. Este indicador, apesar de ser bastante utilizado em estudos epidemiológicos, revela uma margem de erro elevada, principalmente quando utilizado com indivíduos que praticam exercício físico frequentemente. Apesar de todas as lacunas deste parâmetro e estando nós conscientes delas optámos pela sua utilização visto ser um indicador utilizado em inúmeros estudos deste género, sendo mais um indicador a ter em consideração.

Os aspetos que acabámos de referir são, naturalmente, apenas alguns, conscientes de que imensas perguntas sem resposta vagueiam neste preciso instante pela mente de variadíssimos investigadores e outros profissionais que têm a necessidade de, para além da imensa informação científica sobre esta matéria, poderem melhorar a cientificidade da temática em causa.

\section{CONCLUSÕES}

Os resultados evidenciam que, na comparação entre os dois países, os alunos Italianos apresentam resultados mais favoráveis nos indicadores de composição corporal (IMC e PC) avaliados, já para os valores espirométricos mensurados, os alunos Portugueses apresentam resultados mais elevados.

Verificamos ainda que, tanto os alunos Portugueses, como os alunos Italianos que praticam exercício supervisionado, regular e com intensidade planificada, apresentam valores mais favoráveis a um bom estado de saúde comparativamente aos alunos sedentários.

Os resultados parecem evidenciar que as atividades físicas supervisionadas e com objetivos quanto à intensidade e tipo de exercício, de forma continuada e regular, consolidam uma melhoria na composição corporal e na funcionalidade pulmonar, comparativamente a alunos sedentários que recorrem sistematicamente a estilos de vida pouco ativos, independentemente do país. Estes indicadores evidenciam que estilos de vida sedentários podem causar alterações negativas nos valores de composição corporal e nos valores espirométricos, limitadores da funcionalidade do dia-a-dia e, possivelmente, também da prática de atividades físicas. Com base nestes indicadores, cremos ser de importância considerável a criação de mais estratégias e incentivos para a prática de atividades 
físicas supervisionadas nas instituições de Ensino Superior e, quem sabe, proporcionar a introdução de unidades curriculares de cariz prático, com exercício supervisionado, nos planos de estudos de todos os cursos deste nível de ensino, independentemente da área de formação, podendo estas ser optativas.

\section{Agradecimentos:}

A todos os que colaboraram na fase de recolha e tratamento de dados. Ao Instituto Politécnico de Castelo Branco e Universidade da Beira Interior pelo incentivo e pela aquisição de algum equipamento.

\section{Conflito de Interesses:}

Nada a declarar.

\section{Financiamento:}

Nada a declarar.

\section{REFERÊNCIAS}

Callaway, C. W., Chumlea, W. C., Bouchard, C., Himes, J. H., Lohman, T. G., Martin, A. D., ... Seefeldt, V. D. (1988). Circumferences. Em T. G. Lohman, A. F. Roche, \& R. Martorell (Eds.), Anthropometric Standardization Reference Manual (pp. 39-54). Champaign, Illinois: Human Kinetics.

Chen, Y., Horne, S. L., \& Dosman, J. A. (1993). Body weight and weight gain related to pulmonary function decline in adults: a six year follow up study. Thorax, 48(4), 375-380.

Chinn, S. (2005). Concurrent trends in asthma and obesity. Thorax, 60(1), 3-4. http://doi.org/10.1136/thx.2004.031161

Collins, L. C., Hoberty, P. D., Walker, J. F., Fletcher, E. C., \& Peiris, A. N. (1995). The effect of body fat distribution on pulmonary function tests. Chest, 107(5), 1298-1302.

Declaração de Helsínquia. (1975). Princípios Éticos para Pesquisa Clínica Envolvendo Seres Humanos (Assembleia Geral da Associação Médica Mundial, Helsínquia). Finlândia.

De Sá Pinto, A. L., de Barros Holanda, P. M., Radu, A. S., Villares, S. M. F., \& Lima, F. R. (2006). Musculoskeletal findings in obese children. Journal of Paediatrics and Child Health, 42(6), 341-344. http://doi.org/10.1111/j.14401754.2006.00869.x
Dias, D. F., Reis, I. C. B. dos, Reis, D. A. dos, Cyrino, E. S., Ohara, D., Carvalho, F. O., ... Loch, M. R. (2008). Comparison of the health related physical fitness of adults of differents ages. Brazilian Journal of Kinanthropometry and Human Performance, 10(2), 123-128.

Dockery, D. W., Berkey, C. S., Ware, J. H., Speizer, F. E., \& Ferris, B. G. (1983). Distribution of forced vital capacity and forced expiratory volume in one second in children 6 to 11 years of age. The American Review of Respiratory Disease, 128(3), 405-412.

Fung, K. P., Lau, S. P., Chow, O. K., Lee, J., \& Wong, T. W. (1990). Effects of overweight on lung function. Archives of Disease in Childhood, 65(5), 512-515.

Gordon, C. C., Chumlea, W. C., \& Roche, A. F. (1991). Stature, recumbent length, and weight. Em T. G. Lohman \& R. Martorel (Eds.), Anthropometric Standardization Reference Manual (pp. 3-8). Champagn, IL: Human Kinetics Books.

He, Q., Wong, T., Du, L., Jiang, Z., Qiu, H., Gao, Y., ... Yu, I. T. (2009). Respiratory health in overweight and obese Chinese children. Pediatric Pulmonology, 44(10), 997-1002. http://doi.org/10.1002/ppul.21091

Inselma, L. S., Milanese, A., \& Deurloo, A. (1993). Effect of obesity on pulmonary function in children. Pediatric Pulmonology, 16(2), 130-137. http://doi.org/10.1002/ppul.1950160209

Lazarus, R., Sparrow, D., \& Weiss, S. T. (1997). Effects of obesity and fat distribution on ventilatory function: the normative aging study. Chest, 111(4), 891-898.

McArdle, W. D., Katch, F. I., \& Katch, V. L. (2011). Fisiologia do Exercício: Energia, Nutrição e Desempenho Humano ( $7^{\text {a }}$ ed.). Rio de Janeiro: Guanabara Koogan.

Miller, M. R., Hankinson, J., Brusasco, V., Burgos, F., Casaburi, R., Coates, A., ... ATS/ERS Task Force. (2005). Standardisation of spirometry. The European Respiratory Journal, 26(2), 319338. http://doi.org/10.1183/09031936.05.0003480 5

Padez, C., Fernandes, T., Mourão, I., Moreira, P., \& Rosado, V. (2004). Prevalence of overweight and obesity in 7-9-year-old Portuguese children: trends in body mass index from 1970-2002. American Journal of Human Biology, 16(6), 670-678. http://doi.org/10.1002/ajhb.20080

Paulo, R., Petrica, J., \& Martins, J. (2013). Physical activity and respiratory function: corporal composition and spirometric values analysis. Acta Médica Portuguesa, 26(3), 258-264.

Pereira, R. A., Sichieri, R., \& Marins, V. M. (1999). Razão cintura/quadril como preditor de hipertensão arterial. Cadernos de Saúde Pública, 15(2), 333-344. 
Rizzo, N. S., Ruiz, J. R., Hurtig-Wennlöf, A., Ortega, F. B., \& Sjöström, M. (2007). Relationship of physical activity, fitness, and fatness with clustered metabolic risk in children and adolescents: the European youth heart study. The Journal of Pediatrics, 150(4), 388-394. http://doi.org/10.1016/j.jpeds.2006.12.039

Rodrigues, J. C., Cardieri, J. M., Bussamra, M. H., Nakaie, C. M., Almeida, M. B., Silva Filho, L. V., \& Adde, F. V. (2002). Provas de função pulmonar em crianças e adolescentes. Jornal de Pneumologia, 28(Supl.3), 207-221.

Rubinstein, I., Zamel, N., DuBarry, L., \& Hoffstein, V. (1990). Airflow limitation in morbidly obese, nonsmoking men. Annals of Internal Medicine, 112(11), 828-832.

Sahebjami, H. (1998). Dyspnea in obese healthy men. Chest, 114(5), 1373-1377.

Salas, T., Rubies, C., Gallego, C., Muñoz, P., Burgos, F., \& Escarrabill, J. (2011). Technical requirements of spirometers in the strategy for guaranteeing the access to quality spirometry. Archivos de Bronconeumología, 47(9), 466-469. http://doi.org/10.1016/j.arbres.2011.06.005

Silva, Á. M. O., Boin, I. F. S., Pareja, J. C., \& Magna, L. A. (2007). Análise da função respiratória em pacientes obesos submetidos à operação Fobi-
Capella. Revista do Colégio Brasileiro de Cirurgiões, 34(5), 314-320. http://doi.org/10.1590/S010069912007000500007

Slentz, C. A., Duscha, B. D., Johnson, J. L., Ketchum, K., Aiken, L. B., Samsa, G. P., ... Kraus, W. E. (2004). Effects of the amount of exercise on body weight, body composition, and measures of central obesity: STRRIDE--a randomized controlled study. Archives of Internal Medicine, 164(1), 31-39. http://doi.org/10.1001/archinte.164.1.31

Sue, D. Y. (1997). Obesity and pulmonary function: more or less? Chest, 111(4), 844-845.

Telama, R., Yang, X., Laakso, L., \& Viikari, J. (1997). Physical activity in childhood and adolescence as predictor of physical activity in young adulthood. American Journal of Preventive Medicine, 13(4), 317-323.

World Health Organization. (1998). Obesity Status: preventing and managing the global epidemic. (Report of a WHO Consultation on Obesity). Geneva.

Zerah, F., Harf, A., Perlemuter, L., Lorino, H., Lorino, A. M., \& Atlan, G. (1993). Effects of obesity on respiratory resistance. Chest, 103(5), 14701476.

Todo o conteúdo da revista Motricidade está licenciado sob a Creative Commons, exceto quando especificado em contrário e nos conteúdos retirados de outras fontes bibliográficas. 